\title{
TRIPODS/MOPTA 2018 special issue on energy and optimization
}

\author{
Daniel Bienstock ${ }^{1} \cdot$ Luis F. Zuluaga $^{2}$
}

Received: 28 April 2020 / Revised: 28 April 2020 / Accepted: 28 April 2020 / Published online: 2 May 2020 (c) Springer Science+Business Media, LLC, part of Springer Nature 2020

This Optimization and Engineering (OPTE) special issue on Energy and Optimization contains a selection of papers that were presented at the TRIPODS/MOPTA conference held at Lehigh University, Bethlehem, Pennsylvania, in August 15-17, 2018, with funding from the NSF Transdisciplinary Research in Principles of Data Science (TRIPODS) program. MOPTA is an annual confenrece hosted by the Industrial and Systems Engineering Department, Lehigh University, that is aimed to bring together a diverse group of researchers and practitioners, working on both theoretical and practical aspects of continuous or discrete optimization (see https://coral.ise. lehigh.edu/ mopta/). The goal is to host presentations on exciting developments in different areas of optimization and, at the same time, provide a setting for close interaction among the participants. The three papers contained in this volume represent some of the novel ideas in the area of Energy and Optimization discussed at the conference.

Before discussing the articles, it is worth emphasizing the enormous importance of optimization in the area of energy. Rapid and compelling changes in the energy sector have created a wide range of challenges that are being addressed by a combination of engineering and optimization techniques. For example, consider: the use of different optimization techniques to obtain optimal or near-optimal solutions to the alternating current optimal power flow (ACOPF) problem (see, e.g., Kim and Wright 2016; Jabr 2003; Ghaddar et al. 2015; Low 2014), or for gas (see, e.g., Wu et al. 2007; Borraz-Sánchez et al. 2016) and water (see. e.g., Bragalli et al. 2012) distribution network problems; the use of multi-level optimization to address commodity pricing problems (see, e.g., Fampa et al. 2008) or to immunize a commodity network against potential attacks (see, e.g., Arroyo and Galiana 2005); the use of

Luis F. Zuluaga

luis.zuluaga@lehigh.edu

Daniel Bienstock

dano@ieor.columbia.edu

1 Industrial Engineering and Operations Research/Applied Mathematics, Electrical Engineering, Columbia University, New York City, NY, USA

2 Industrial and Systems Engineering, Lehigh University, Bethlehem, PA, USA 
uncertainty optimization techniques to address challenges related to the integration of renewable energy sources (RES) into electricity markets (see, e.g., Jabr 2013), immunize commodity networks against catastrophic events (see, e.g., Pinar et al. 2010), and obtain robust equilibrium commodity prices that take into account uncertainty in commodity production or demand (see, e.g., Morales et al. 2012; Nowak et al. 2005); the development of improved mathematical formulations and optimization solution methods, to address large-scale energy related problems (see, e.g., Pruitt et al. 2014; Leuthold et al. 2012); among many other interesting research directions. The overall goal of this research is the design of commodity networks that are "smart", interconnected, efficient, reliable, resilient, and environmentally responsible. Beyond the study of individual commodity networks, very interesting current research addresses the challenging problem of studying the interaction between different networks such as electricity, gas, and water networks, as well as the interaction of these networks with communication and transportation systems.

In line with these important and novel research developments, Eltved, Dahl, and Andersen show in "On the Robustness and Scalability of Semidefinite Relaxation for Optimal Power Flow Problems" (Dahl et al. 2020) how semidefinite programming (SDP) relaxations of the ACOPF problem, which have been shown to be very tight, can be effectively solved for large-scale electricity networks of up to 82,000 buses. The authors' results rely on an appropriate reformulation and scaling of the SDP relaxation of the ACOPF problem.

In "Stochastic Hydro-thermal Unit Commitment via Multi-level Scenario Trees and Bundle Regularization", Finardi, Lobato, de Matos, and Sagastizábal (Sagastizábal et al. 2020) propose a novel strategy to model uncertainty in unit commitment problems; namely the use of multi-horizon scenario trees. This strategy, in combination with a regularized Benders decomposition approach, allows to effectively solve realistic hydro-thermal unit commitment problems.

Finally, in "Unified Value-based Feedback, Optimization and Risk Resource Management in Complex Electric Energy Systems", Ilic and Jaddivada (2020) consider a complex interconnected electric energy system with components having highly heterogeneous internal dynamics. To effectively model this problem, an inherent multi-layered structure is utilized by expressing interconnection constraints in terms of unified power of variables and their dynamics. Among other results in this paper, the authors present a method to allow decision makers in a distributed system to create a bid function for the purpose of coordinating market-clearing.

\section{References}

Arroyo JM, Galiana FD (2005) On the solution of the bilevel programming formulation of the terrorist threat problem. IEEE Trans Power Syst 20(2):789-797

Borraz-Sánchez C, Bent R, Backhaus S, Hijazi H, Hentenryck PV (2016) Convex relaxations for gas expansion planning. INFORMS J Comput 28(4):645-656

Bragalli C, D'Ambrosio C, Lee J, Lodi A, Toth P (2012) On the optimal design of water distribution networks: a practical minlp approach. Optim Eng 13(2):219-246 
Dahl J, Andersen MS, Eltved A (2020) On the robustness and scalability of semidefinite relaxation for optimal power flow problems. Optim Eng 21(2):375-392

Fampa M, Barroso L, Candal D, Simonetti L (2008) Bilevel optimization applied to strategic pricing in competitive electricity markets. Comput Optim Appl 39(2):121-142

Ghaddar B, Marecek J, Mevissen M (2015) Optimal power flow as a polynomial optimization problem. IEEE Trans Power Syst 31(1):539-546

Ilic M, Jaddivada R (2020) Unified value-based feedback, optimization and risk management in complex electric energy systems. Optim Eng 21(2):427-483

Jabr RA (2003) A primal-dual interior-point method to solve the optimal power flow dispatching problem. Optim Eng 4(4):309-336

Jabr RA (2013) Adjustable robust opf with renewable energy sources. IEEE Trans Power Syst 28(4):4742-4751

Kim T, Wright SJ (2016) An S $\ell_{1}$ LP-active set approach for feasibility restoration in power systems. Optim Eng 17(2):385-419

Leuthold FU, Weigt H, von Hirschhausen C (2012) A large-scale spatial optimization model of the european electricity market. Netw Spatial Econ 12(1):75-107

Low SH (2014) Convex relaxation of optimal power flow?Part I: formulations and equivalence. IEEE Trans Control Netw Syst 1(1):15-27

Morales JM, Conejo AJ, Liu K, Zhong J (2012) Pricing electricity in pools with wind producers. IEEE Trans Power Syst 27(3):1366-1376

Nowak MP, Schultz R, Westphalen M (2005) A stochastic integer programming model for incorporating day-ahead trading of electricity into hydro-thermal unit commitment. Optim Eng 6(2):163-176

Pinar A, Meza J, Donde V, Lesieutre B (2010) Optimization strategies for the vulnerability analysis of the electric power grid. SIAM J Optim 20(4):1786-1810

Pruitt KA, Leyffer S, Newman AM, Braun RJ (2014) A mixed-integer nonlinear program for the optimal design and dispatch of distributed generation systems. Optim Eng 15(1):167-197

Sagastizábal C, Finardi E, Lobato R, de Matos V, Tomasgard A (2020) Stochastic hydro-thermal unit commitment via multi-level scenario trees and bundle regularization. Optim Eng 21(2):393-426

Wu Y, Lai KK, Liu Y (2007) Deterministic global optimization approach to steady-state distribution gas pipeline networks. Optim Eng 8(3):259-275

Publisher's Note Springer Nature remains neutral with regard to jurisdictional claims in published maps and institutional affiliations. 